Highly sensitive determination of 68 psychoactive pharmaceuticals, illicit drugs, and related human metabolites in wastewater by liquid chromatographytandem mass spectrometry

Viola L. Borova, Niki C. Maragou, Pablo Gago-Ferrero, Constantinos Pistos \& Nikolaos S. Thomaidis

\section{Analytical and Bioanalytical Chemistry}

ISSN 1618-2642

Volume 406

Number 17

Anal Bioanal Chem (2014)

406:4273-4285

DOI 10.1007/s00216-014-7819-3
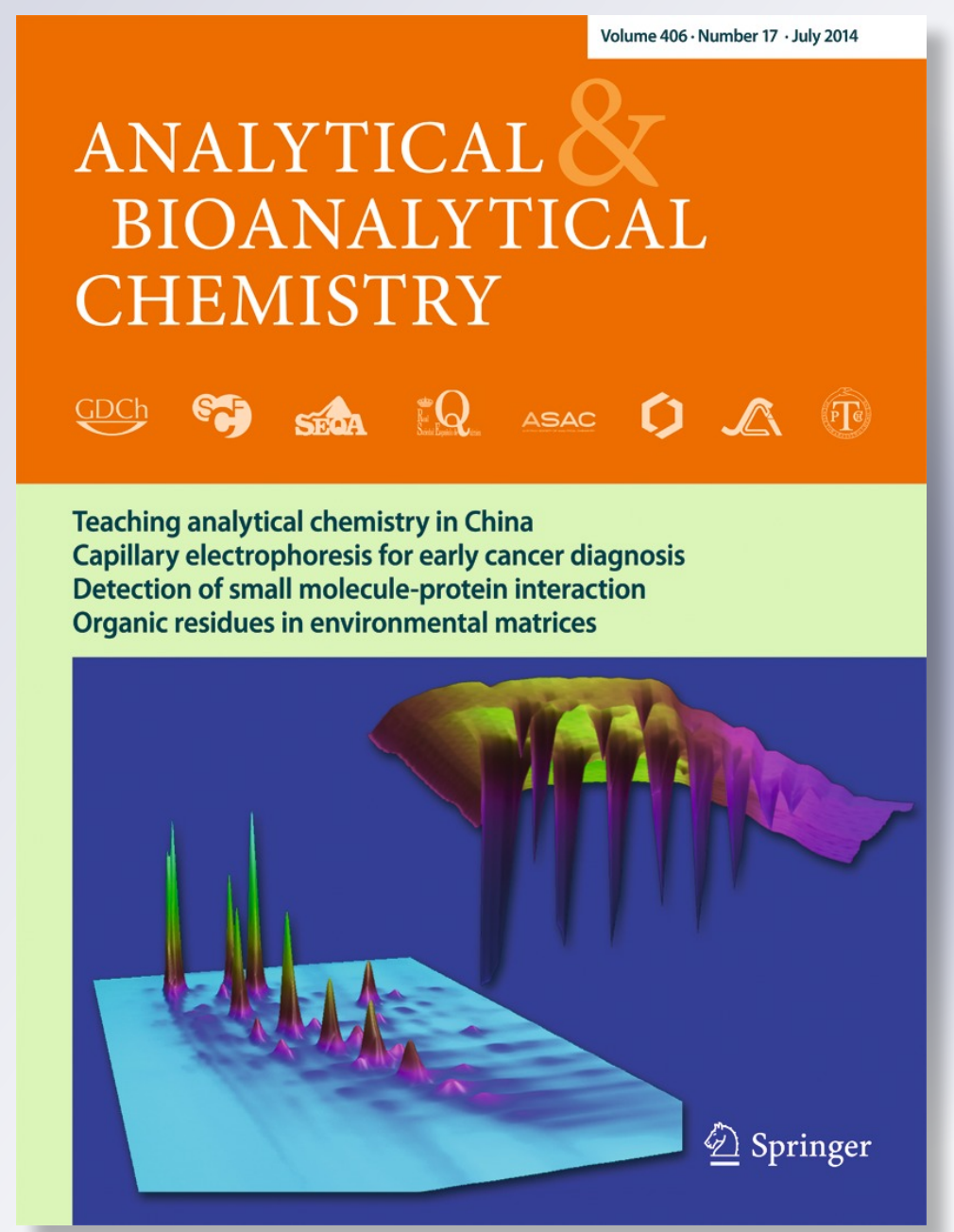

Springer 
Your article is protected by copyright and all rights are held exclusively by SpringerVerlag Berlin Heidelberg. This e-offprint is for personal use only and shall not be selfarchived in electronic repositories. If you wish to self-archive your article, please use the accepted manuscript version for posting on your own website. You may further deposit the accepted manuscript version in any repository, provided it is only made publicly available 12 months after official publication or later and provided acknowledgement is given to the original source of publication and a link is inserted to the published article on Springer's website. The link must be accompanied by the following text: "The final publication is available at link.springer.com". 


\title{
Highly sensitive determination of 68 psychoactive pharmaceuticals, illicit drugs, and related human metabolites in wastewater by liquid chromatography-tandem mass spectrometry
}

\author{
Viola L. Borova • Niki C. Maragou • \\ Pablo Gago-Ferrero • Constantinos Pistos • \\ Nikolaos S. Thomaidis
}

Received: 20 January 2014 /Revised: 25 March 2014 / Accepted: 4 April 2014 / Published online: 2 May 2014

(C) Springer-Verlag Berlin Heidelberg 2014

\begin{abstract}
The present work describes the development and validation of a highly sensitive analytical method for the simultaneous determination of 68 compounds, including illicit drugs (opiates, opioids, cocaine compounds, amphetamines, and hallucinogens), psychiatric drugs (benzodiazepines, barbiturates, anesthetics, antiepileptics, antipsychotics, antidepressants, and sympathomimetics), and selected human metabolites in influent and effluent wastewater (IWW and EWW) by liquid chromatography coupled to tandem mass spectrometry (LC-MS/MS). The method involves a pre-concentration and cleanup step, carried out by solid-phase extraction (SPE) using the adsorbent Strata-XC, followed by the instrumental analysis performed by LC-MS/MS, using a Kinetex pentafluorophenyl (PFP) reversed-phase fused-core column and electrospray ionization (ESI) in both positive and negative modes. A systematic optimization of mobile phases was performed to cope with the wide range of physicochemical properties of the analytes. The PFP column was also compared with two reversed-phase columns: fused-core C18 and XBC18 (with a cross-butyl C18 ligand). SPE optimization and
\end{abstract}

Electronic supplementary material The online version of this article (doi:10.1007/s00216-014-7819-3) contains supplementary material, which is available to authorized users.

V. L. Borova · N. C. Maragou • P. Gago-Ferrero •

N. S. Thomaidis $(\square)$

Laboratory of Analytical Chemistry, Department of Chemistry,

National and Kapodistrian University of Athens, Panepistimiopolis, Zografou, 15771 Athens, Greece

e-mail: ntho@chem.uoa.gr

C. Pistos

Laboratory of Forensic Medicine and Toxicology, School of Medicine, National and Kapodistrian University of Athens, 15771 Athens, Greece critical aspects associated with the trace level determination of the target compounds (e.g., matrix effects) have been also considered and discussed. Fragmentation patterns for all the classes were proposed. The validated method provides absolute recoveries between 75 and $120 \%$ for most compounds in IWW and EWW. Low method limits of detection were achieved (between 0.04 and $10.0 \mathrm{ng} / \mathrm{L}$ for $87 \%$ of the compounds), allowing a reliable and accurate quantification of the analytes at trace level. The method was successfully applied to the analysis of these compounds in five wastewater treatment plants in Santorini, a touristic island of the Aegean Sea, Greece. Thirty-two out of 68 compounds were detected in all IWW samples in the range between $0.6 \mathrm{ng} / \mathrm{L}$ (for nordiazepam) and $6,822 \mathrm{ng} / \mathrm{L}$ (for carbamazepine) and 22 out of 68 in all EWW samples, with values between $0.4 \mathrm{ng} /$ $\mathrm{L}$ (for 9-OH risperidone) and 2,200 ng/L (for carbamazepine). The novel methodology described herein maximizes the information on the environmental analysis of these substances and also provides a first profile of 68 drugs in a Greek touristic area.

Keywords Illicit drugs · Psychiatric drugs - Wastewater . LC-MS/MS · SPE

\section{Introduction}

From the first import into Europe of cannabis as a therapeutic drug, in the nineteenth century by O'Shaugnessy (1838-1839) [1], until the present time, the usage profile of psychotropic and illicit drugs has changed dramatically. The progress of medical science, the great development of chemistry and pharmaceutical companies, and also the changes in social conditions have led to a large growth of the number and use 
of psychotropic substances. According to the European Monitoring Centre for Drugs and Drug Addiction (EMCDDA 2012) estimations [2], 42.5 million Europeans (between 15 and 34 years old) had smoked cannabis at least once ( $10.8 \%$ in Greece), 8 million had consumed cocaine (1.0\% in Greece), 7.0 million had taken amphetamines ( $0.2 \%$ in Greece), and 7.5 million had used ecstasy $(0.6 \%$ in Greece). The consumption of psychiatric drugs has also increased a lot during the last years as a consequence of the increased financial European crisis, which can lead to psychological health effects causing several psychiatric diseases [3, 4]. Psychiatric and illicit drugs have become pseudo-persistent in the environment due to their high volumes of production and use, and nowadays, they are considered environmental emerging contaminants [5-7]. Following consumption, these compounds and their metabolites are continuously discharged into wastewaters due to human excretion after legal or illegal consumption or occasional direct disposal of clandestine laboratory wastes into sewage systems $[8,9]$. These substances and related metabolites (since they are partly metabolized) are continuously released into the aquatic environment through effluent wastewaters (EWW) due to their partial elimination in sewage treatment plants $[6-8,10,11]$. These compounds are biologically active and have been designed to exert specific effects on organisms. Despite low concentrations, the effects of these substances in the environment and human health cannot be excluded. Substances such as cocaine, morphine, or various amphetamines such as ecstasy $(3,4$ methylenedioxy- $N$-methylamphetamine (MDMA)) have powerful pharmacological effects, and their presence as mixtures in superficial waters may cause toxic effects on aquatic organisms $[7,10,12,13]$. Substances like the selective serotonin reuptake inhibitors (SSRIs) and serotonin-norepinephrine reuptake inhibitors (SNRIs), venlafaxine, paroxetine, and fluoxetine, with a high consumption rate along the population of Europe, are listed in the top-ten list of the most harmful psychoactive drugs [14]. Moreover, recent results also showed clear cyto-genotoxic effects of some drugs (e.g., cocaine) in common nontarget organism, highlighting the risk of illicit drugs in the ecosystem [15].

Different groups of illicit, stimulant, and psychiatric drugs, including opiates and opioids, hallucinogens, barbiturates, antipsychotics, sedatives, or antidepressants, have been detected in urban wastewater and surface water (lake and river water) from Germany [16], Spain [17-29], USA and Canada [8, 9, 30-32], France [33], Ireland [34], Belgium [6, 35, 36], UK [37-39], Netherlands [40] Italy, and Switzerland [10-12, $41,42]$.

In recent years, several authors have developed analytical methodologies to determine psychoactive pharmaceuticals, illicit drugs, and their metabolites in superficial water and wastewater with the objective of monitoring their environmental occurrence and also to estimate drug usage at the community level. Liquid chromatography coupled to tandem mass spectrometry (LC-MS/MS) is the most used technique for the analysis of these substances in the aquatic environment due to its versatility, specificity, and selectivity $[6,10-12$, 16-23, 25-44]. Most of these methodologies used C18 reversed-phase column, and only one among them used a pentafluorophenyl (PFP) revered-phase column [43] providing results just for a small number of polar compounds. The PFP stationary phase incorporates fluorine atoms on the periphery of the phenyl ring to provide a unique aromatic and polar selectivity.

The main objective pursued within the study was to develop and validate a multi-analyte method for the simultaneous determination of 68 different psychoactive pharmaceuticals and illicit drugs and their metabolites (a particular mixture of compounds with very different physicochemical properties) in municipal wastewaters using a PFP column for their separation. Compromised LC-electrospray ionization (ESI)-MS/ MS conditions for the 68 target compounds were provided, as well as proposed fragmentation patterns for all compounds. The main difficulties of this study include the wide range of polarities of the compounds, the zwitterionic character, and different chemical characteristics [45] and also the multi-trace concentration levels that are usually detected. To overcome these difficulties, a rigorous optimization of sample preparation, mobile and stationary phases, and solid-phase extraction (SPE) sorbents as well as ESI-MS condition optimizations were performed. The developed methodology was applied for the determination, for the first time in Greece, of the presence of psychoactive pharmaceuticals, illicit drugs, and their metabolites in wastewater. The occurrence of these substances was evaluated in five wastewater treatment plants (WWTPs) located in Santorini, a touristic island of the Aegean Sea.

\section{Materials and methods}

\section{Chemicals and reagents}

Up to 68 drugs and metabolites, belonging to opiates, opioids and their metabolites (7), cocaine and metabolites (3), amphetamines (5), hallucinogens (cannabinoids (2)), lysergic acid diethylamide (LSD) (2), benzodiazepines (13), barbiturates (2), anesthetics (6), antiepileptics (7), antipsychotics (6), antidepressants (tricyclic (5), tetracyclic (2)), SSRIs (5), SNRIs (1), and sympathomimetics (2) were determined by LC-ESI-MS/MS. Some features of the studied compounds including analyte name, Chemical Abstracts Service number, chemical structure, molecular formula, molecular weight, $\mathrm{pKa}$ and $\log -K_{\mathrm{ow}}$ are shown in Electronic Supplementary Material Table S1.

High-purity individual standards ( $>98 \%$ ), solutions or solids, of all the target analytes were purchased from LGC 
Promochem (Molsheim, France) except topiramate and lamotrigine, which were obtained from Glenmark (Mahwah, NJ, USA) and Sigma Aldrich Chemie GmbH (Steinheim, Germany), respectively. All deuterated compounds were also obtained from LGC Promochem (Molsheim, France): morphine-D3 (MOR-D3), codeine-D3 (COD-D3), cocaineD3 (COC-D3), 2-ethylidene-1,5-dimethyl-3,3diphenylpyrrolidine-D3 (EDDP-D3), ecgonine methyl esterD3 (EME-D3), 3,4-methylenedioxy- $N$-methylamphetamineD5 (MDMA-D5), 3,4-methylenedioxy amphetamine-D5 (MDA-D5), tetrahydrocannabinol-D3 (THC-D3), tetrahydrocannabinolic acid-D3 (THCA-D3), and lysergic acid diethylamide-D3 (LSD-D3). Individual stock solutions were prepared in either acetone or methanol $(\mathrm{MeOH})$, at concentrations varying between 10 and $1,250 \mathrm{mg} / \mathrm{L}$. Solutions for direct injection (infusion) of individual standards and internal standards (IS) were prepared at concentrations of $2 \mathrm{mg} / \mathrm{L}$ in acetonitrile $(\mathrm{ACN}) /$ water $(50: 50, v / v)$, just before direct injection experiments. Working solutions were prepared daily by appropriate dilution of the mixture stock standard and IS solutions in $\mathrm{MeOH}$.

Calibration standards were prepared by serial dilution of the mixed working solution using Milli-Q water resulting in individual concentrations ranging from 1 to $100 \mu \mathrm{g} / \mathrm{L}$ All stock and working solutions were stored in glass bottles in the dark at $-20{ }^{\circ} \mathrm{C}$. MeOH and ACN (both of LC-MS quality, $99.9 \%$ purity, Lichrosolv), were purchased from Merck (Darmstadt, Germany). High-purity water was prepared using a Milli-Q water purification system (Millipore Direct-Q UV, Bedford, MA, USA). Formic acid (98\% purity, HPLC grade) was obtained from Sigma-Aldrich (Fluka, Germany). Hydrochloric acid ( $\mathrm{HCl})(37 \%)$ was purchased from Merck (Darmstadt, Germany), and ammonium hydroxide was prepared using ammonia (25\%), which was purchased from Panreac (Barcelona, Spain). Strata-X $(200 \mathrm{mg} / 6 \mathrm{~mL})$ and Strata-XC (200 mg/6 mL) cartridges and syringe filters (RC) of $4 \mathrm{~mm}$ and with pore size of $0.2 \mu \mathrm{m}$ were obtained from Phenomenex (Torrance, CA, USA). Glass fiber filters (GFF, pore size $0.7 \mu \mathrm{m}$ ) used in wastewater filtration were obtained from Millipore (Cork, Ireland).

\section{Sample collection}

Santorini is an island located in the southern Aegean Sea, about $200 \mathrm{~km}$ southeast from Greece's mainland. It forms the southernmost member of the Cyclades group of islands and covers an area of $73 \mathrm{~km}^{2}$. The census population (data from 2011) is 15,550 inhabitants, but since this island is a very important touristic center, its population increases very considerably during summer periods. Santorini is, along with Anafi Island, the only location in Europe to feature a hot desert climate according to the Köppen climate classification system [46], and most of the water used for human activities comes from desalination plants.

Influent and effluent wastewater samples (grab samples) were collected from five WWTP networks at Santorini Island. Sampling locations are described in detail in Fig. 1. Emporio and Ia are the most populated areas in the island of Santorini, especially during the summer period, when the sampling was carried out (July 2012). The main characteristics of the five WWTP are summarized in Table 1. As the table shows, all the WWTP that were investigated are equipped with conventional activated sludge (CAS) secondary treatment.

Wastewater samples (both IWW and EWW) were collected in plastic (PET) bottles (volume $1.5 \mathrm{~L}$ ) and maintained at chilled conditions until their arrival at the laboratory. After transportation, the samples were directly stored (at $4{ }^{\circ} \mathrm{C}$ ).

\section{Sample pretreatment and solid-phase extraction}

Upon reception in the laboratory, samples were vacuum filtered through GFF. Sample pH was adjusted to 2.5 after filtration using $\mathrm{HCl}(1 \mathrm{M})$ in order to prevent the degradation of the analytes by microorganisms and to facilitate the positive ionization when working with positive electrospray. Internal standards $(50 \mu \mathrm{L}$ of a methanolic mixture containing $1 \mathrm{mg} / \mathrm{L}$ of each deuterated compound) were added to the samples prior to the SPE step in the optimized final method. The extractions of samples were carried out within $24 \mathrm{~h}$ from their arrival in the laboratory and the analysis of extracts, within 7 days after extraction (during this period of days, the extracts were stored in the dark at $-20^{\circ} \mathrm{C}$ ). These storage conditions were chosen based on some studies regarding the stability of the target compounds [47, 48].

In the present study, we compared two different polymeric sorbents, Strata-X (hydrophilic-lipophilic reversed phase) and Strata-XC (strong cation exchange and hydrophilic-lipophilic reversed phase). In the optimized methodology, the Strata-XC polymeric sorbent cartridge $(200 \mathrm{mg} / 6 \mathrm{~mL})$ was selected to perform the SPE step. The SPE procedure was derived from Bisceglia et al. [30] with modifications. Figure 2 shows the SPE procedure with Strata-XC (the optimized methodology) and also with Strata-X (finally discarded). In the optimized method, the cartridges were conditioned with $6 \mathrm{~mL}$ of methanol and $6 \mathrm{~mL}$ of acidified ultrapure water $(\mathrm{pH} 2.5$ with $\mathrm{HCl}$ $1 \mathrm{M})$. Next, 50-mL samples ( $\mathrm{pH} 2.5)$ were loaded under gravity. The cartridges were washed with $3 \mathrm{~mL}$ of ultrapure water $(\mathrm{pH} 2.5)$ and subsequently dried under vacuum for $1 \mathrm{~h}$ and 8 psi. Analytes were eluted with $3 \times 2 \mathrm{~mL}$ of $2 \% \mathrm{NH}_{3}$ in methanol $(\mathrm{pH} \sim 10)$ and, after evaporation to dryness under a constant stream of nitrogen at $40{ }^{\circ} \mathrm{C}$, extracts were reconstituted in $500 \mu \mathrm{L}$ of $54 \% \mathrm{MeOH}$ (aq) followed by a 1 -min vortex stirring. 
Fig. 1 Location of the main urban areas and sampling sites for influent and effluent wastewaters in Santorini

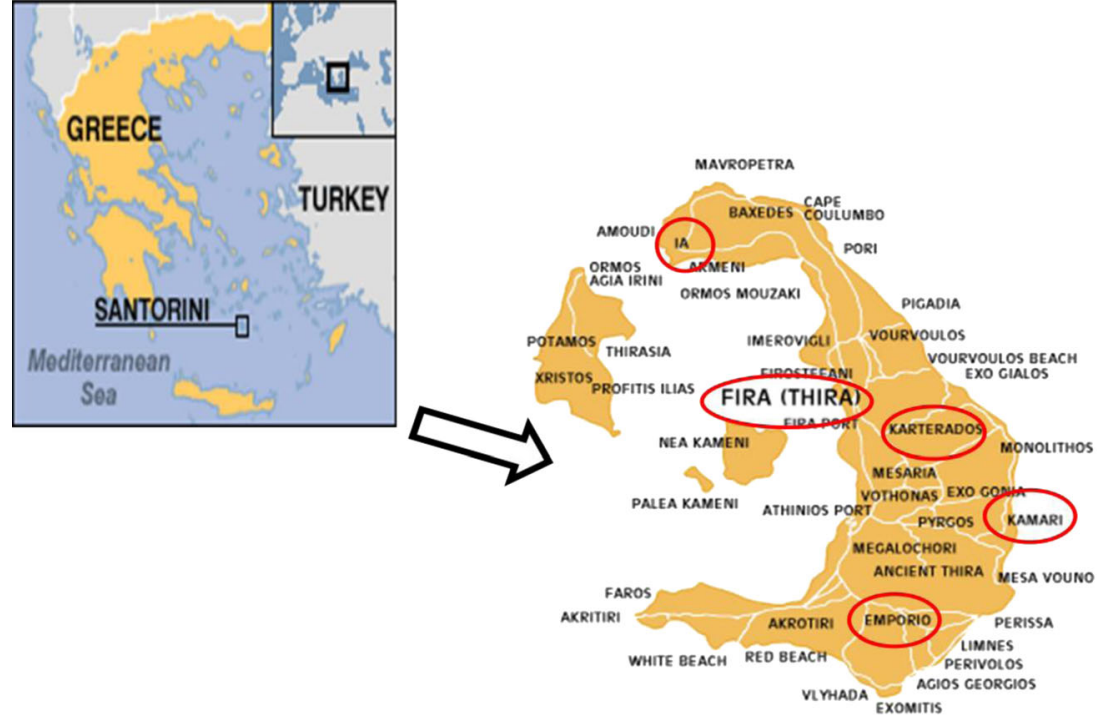

\section{LC-MS/MS analysis}

Chromatographic separation was performed using an Accela gradient UHPLC pump equipped with an Accela Autosampler system from Thermo Electron Corporation (Thermo, San Jose, CA, USA). Separation of the compounds, after mobile phase and column optimization, was achieved on a Kinetex PFP reversed-phase fused-core column $(50 \mathrm{~mm} \times 2.10 \mathrm{~mm}$, $1.7 \mu \mathrm{m})$ equipped with a guard PFP column $(4 \mathrm{~mm} \times 20 \mathrm{~mm})$. In the analysis with electrospray positive ionization mode (PI), the mobile phase was composed of Milli-Q water and $\mathrm{MeOH}$ as organic phase, both containing $0.05 \% v / v$ formic acid. The adopted elution gradient starts with $2 \%$ of $\mathrm{MeOH}$ and keeps constant during $3 \mathrm{~min}$. After that, it increases linearly to $100 \%$ in $20 \mathrm{~min}$. Pure organic conditions were kept constant for $26 \mathrm{~min}$, and finally initial conditions were reached and kept constant for $16 \mathrm{~min}$ for column equilibration. The total run time for each injection was $65 \mathrm{~min}$. The column temperature was kept constant at $25{ }^{\circ} \mathrm{C}$. For the analysis under negative ionization mode (NI), the determination of the analytes was performed also on a Phenomenex ${ }^{\mathrm{TM}}$ Kinetex PFP column. The mobile phase was composed of Milli-Q water and $\mathrm{MeOH}$. The gradient program starts with $30 \%$ of $\mathrm{MeOH}$ for
$3 \mathrm{~min}$, increases linearly to $100 \%$ in $20 \mathrm{~min}$, keeps constant for $7 \mathrm{~min}$, and returns to initial conditions followed by $5 \mathrm{~min}$ of equilibration time. The total run time for each injection was $35 \mathrm{~min}$. The mobile phase flow rate was set to $100 \mu \mathrm{L} / \mathrm{min}$, and the injection volume was $10 \mu \mathrm{L}$ in both ionization modes.

Mass spectrometry analysis was performed with a TSQ Quantum Access triple-quadrupole mass spectrometer from Thermo Electron Corporation (Thermo, San Jose, CA, USA) equipped with an electrospray ionization source (Thermo IonMAX). After optimization, 63 compounds were detected in PI and 5 compounds in NI (Table 2). Identification and quantification were performed under selected-reaction monitoring (SRM) mode. Two characteristic fragments of the precursor molecular ion were monitored for each target analyte. The most abundant transition (precursor/product ion 1) was used for quantification, whereas the second transition (precursor/product ion 2) was used for confirmation. This procedure was in compliance with the European Council Directive 2002/657/EC, that although it was initially conceived for food residue analysis, it has been accepted by the scientific community for environmental analysis. Table 2 shows the fragmentation voltage and collision energies optimized for each transition. For the PI and NI modes, ESI conditions were
Table 1 Characteristics of the wastewater treatment plants (WWTP) investigated

Sampling date: 26 Jul 2012; Primary settling: grinding, desanding and sedimentation; Type of sewage water treated: urban

\begin{tabular}{llllll}
\hline WWTP & $\begin{array}{l}\text { Mean influent flow } \\
\text { rate }\left(\mathrm{m}^{3} / \text { day }\right)\end{array}$ & $\begin{array}{l}\text { Population } \\
\text { served }\end{array}$ & $\begin{array}{l}\text { Average sludge } \\
\text { production }(\mathrm{kg} / \\
\text { day })\end{array}$ & $\begin{array}{l}\text { Primary } \\
\text { treatment }\end{array}$ & $\begin{array}{l}\text { Secondary } \\
\text { treatment }\end{array}$ \\
\hline Kamari & 1,600 & 15,500 & 3,500 & Primary settling & Activated sludge \\
Fira & 1,500 & 10,500 & 2,900 & Primary settling & Activated sludge \\
Karterados & 900 & 3,150 & 580 & Primary settling & Activated sludge \\
Emporio & 600 & 3,000 & 390 & Primary settling & Activated sludge \\
Ia & 700 & 6,000 & 1,000 & Primary settling & Activated sludge \\
\hline
\end{tabular}


Fig. 2 Flow chart of the SPE procedures using both cartridges (Strata-X and Strata-XC)

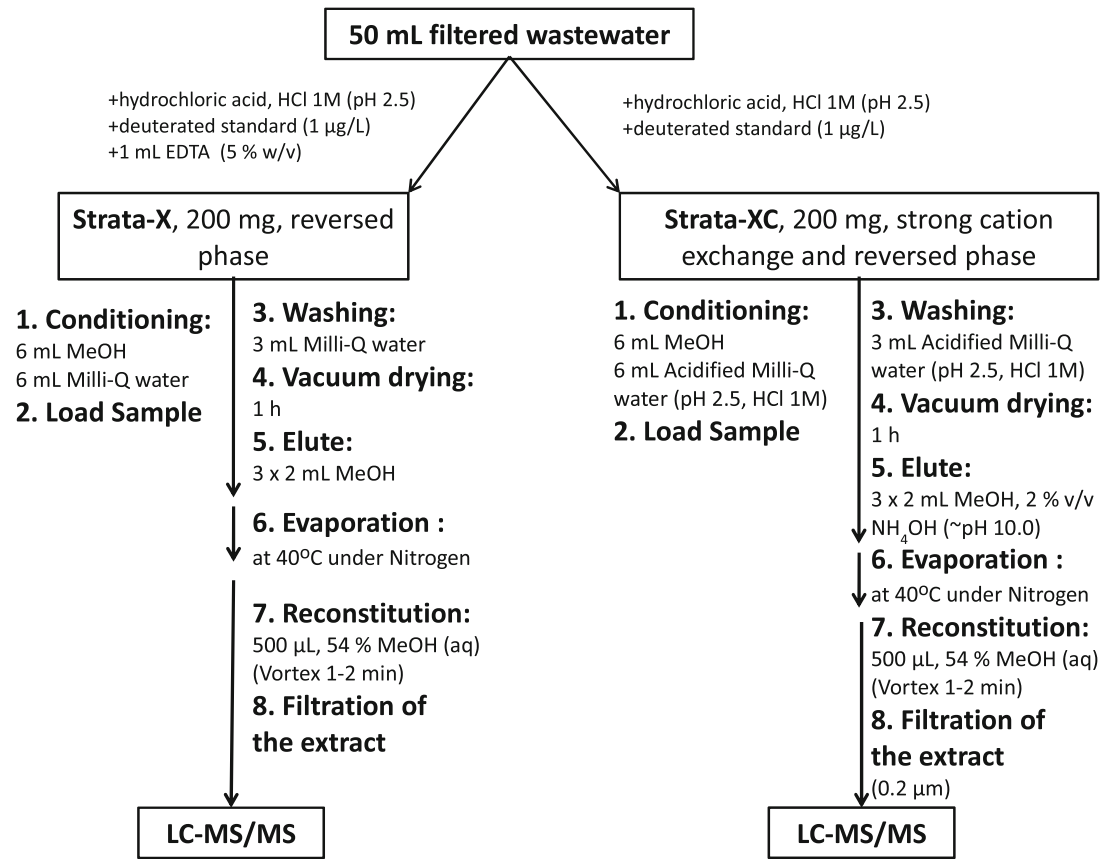

obtained as a compromise using the optimum values for most compounds. A detailed description of optimum MS source conditions as well as the total ion chromatographs in both ionization modes are presented in Electronic Supplementary Material Table S2. Instrument control and data acquisition and evaluation were performed with Excalibur software (Thermo Electron Corporation).

\section{Method validation}

The quantification of compounds in samples was performed using spiked samples with the corresponding labeled analyte. The linearity of the method was studied by analyzing standard solutions in quadruplicate at concentrations ranging from 0.1 to $100 \mu \mathrm{g} / \mathrm{L}$ (seven-point calibration curve) for all the compounds. Standard addition curves were also prepared at the same concentration range. The method limits of detection (MLOD) and the limits of quantification (MLOQ) were calculated by analyzing five times the lowest spiked concentration (for most of the compounds $0.01 \mu \mathrm{g} / \mathrm{L}$ ). For compounds with high concentrations in influents (like codeine, THCA, EME, citalopram, antiepileptics, venlafaxine, ephedrine, or doxepin), replicate analyses of the same sample were performed. MLODs were calculated as follows: the standard deviation of the lowest spiked concentration or the standard deviation of the replicate analyses (for abundant compounds) was multiplied by 3 and then divided by the slope of the calibration curve of spiked samples. The trueness of the method was assessed by spiking experiments at one concentration level, $1.0 \mu \mathrm{g} / \mathrm{L}$ in both influents and effluents. The recovery was calculated by comparing the response in extracted wastewater samples spiked with standard solutions at the same concentration before $(B)$ and after $(A)$ the extraction: REC $(\%)=B / A \times 100$. Overall method repeatability was evaluated by spiking wastewater samples with $1.0 \mu \mathrm{g} / \mathrm{L}$ of each compound $(n=6)$. Matrix effects (ME, $\%$ ) were assessed according to the equation $\operatorname{ME}(\%)=[(A / C)-1] \times 100$, where $C$ is the response obtained in neat standard solutions, and $A$ is the corresponding response for post-extraction spiked samples. Nonspiked samples were measured in parallel, and their signal was subtracted from the spiked ones.

\section{Results and discussion}

\section{SPE procedure}

Sample pre-concentration and purification of the target analytes are critical steps and contribute very significantly to the final performance of the analytical method. Target analytes of the present work show very different physicochemical properties, as it is shown in Electronic Supplementary Material Table S1. The studied drugs are in general basic compounds. The major microspecies at $\mathrm{pH} 2.5$ for most compounds are positively charged [45]. Opiates are characterized by a $\mathrm{NH}^{+}$charged group. Amphetamines, antidepressants, SSRIs, SNRIs, antipsychotics, LSD hallucinogens, and anesthetics (except thiopental, which is not charged) are also positively charged at $\mathrm{pH} 2.5$, with one or two $\mathrm{NH}^{+}$groups. Benzodiazepines at $\mathrm{pH} 2.5$ have both neutral and positive microspecies, since they may accept a proton at the amine group. Oxazepam, lorazepam, and temazepam are an 
焉昜

올

a

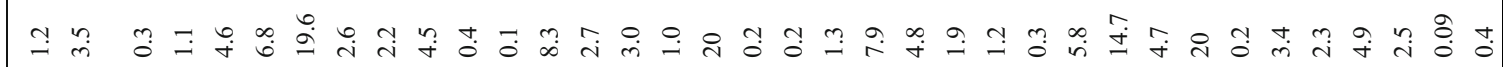

㘳离

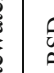

$2 \sum_{0}^{30} 0^{\circ}$ $\varphi+0$ พ 9 $\uparrow \circ \uparrow$ 우 $=$ $n=$ $=$ F 个 ำ $\frac{1}{T}-\frac{1}{1}$ $\underset{1}{\top}$ $\omega_{n} \stackrel{t}{T} \stackrel{4}{\pi}$ $\pm$ $\pm=$ $=$ $\pm \simeq$ ป 2 $\because \simeq$ 6 הุ 范 遂恿 a

$\sum \sum$ के 엉

a

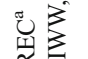

ร.

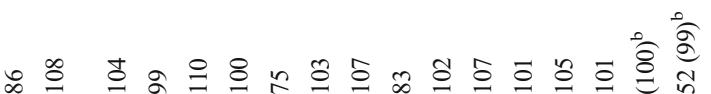
\&

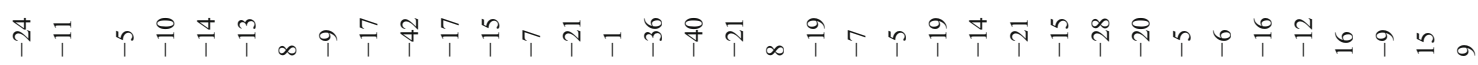

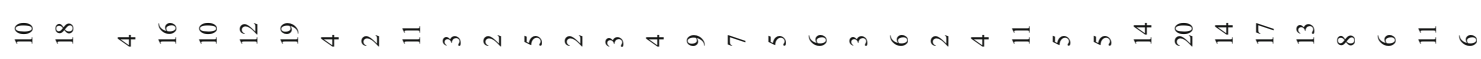
$=0$ 突

=

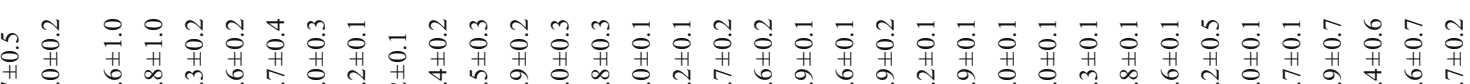
向 e 苍 ․ㅠ 흘 壱 总

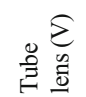
mª

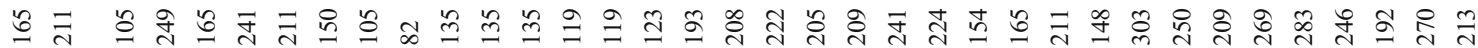

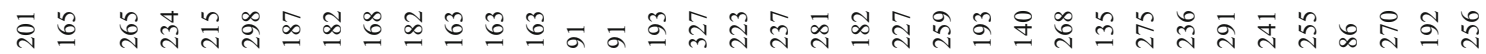

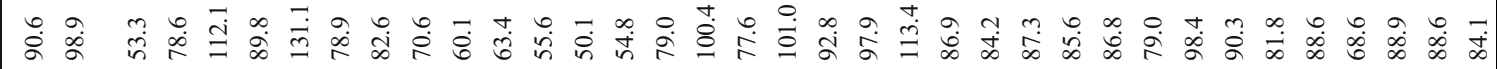

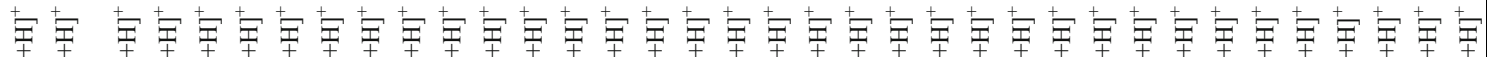

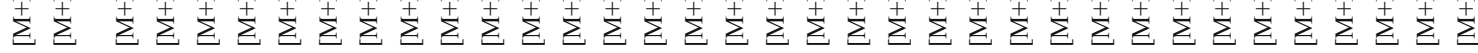
㐫 d
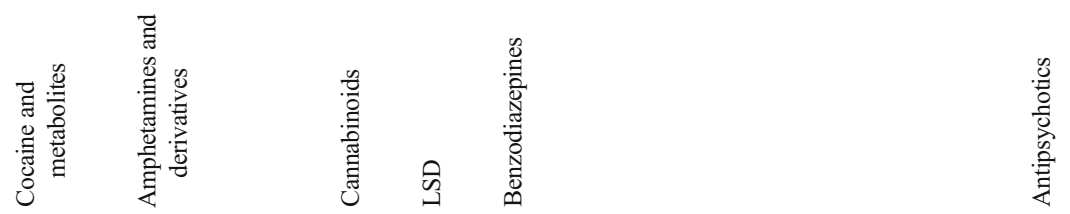


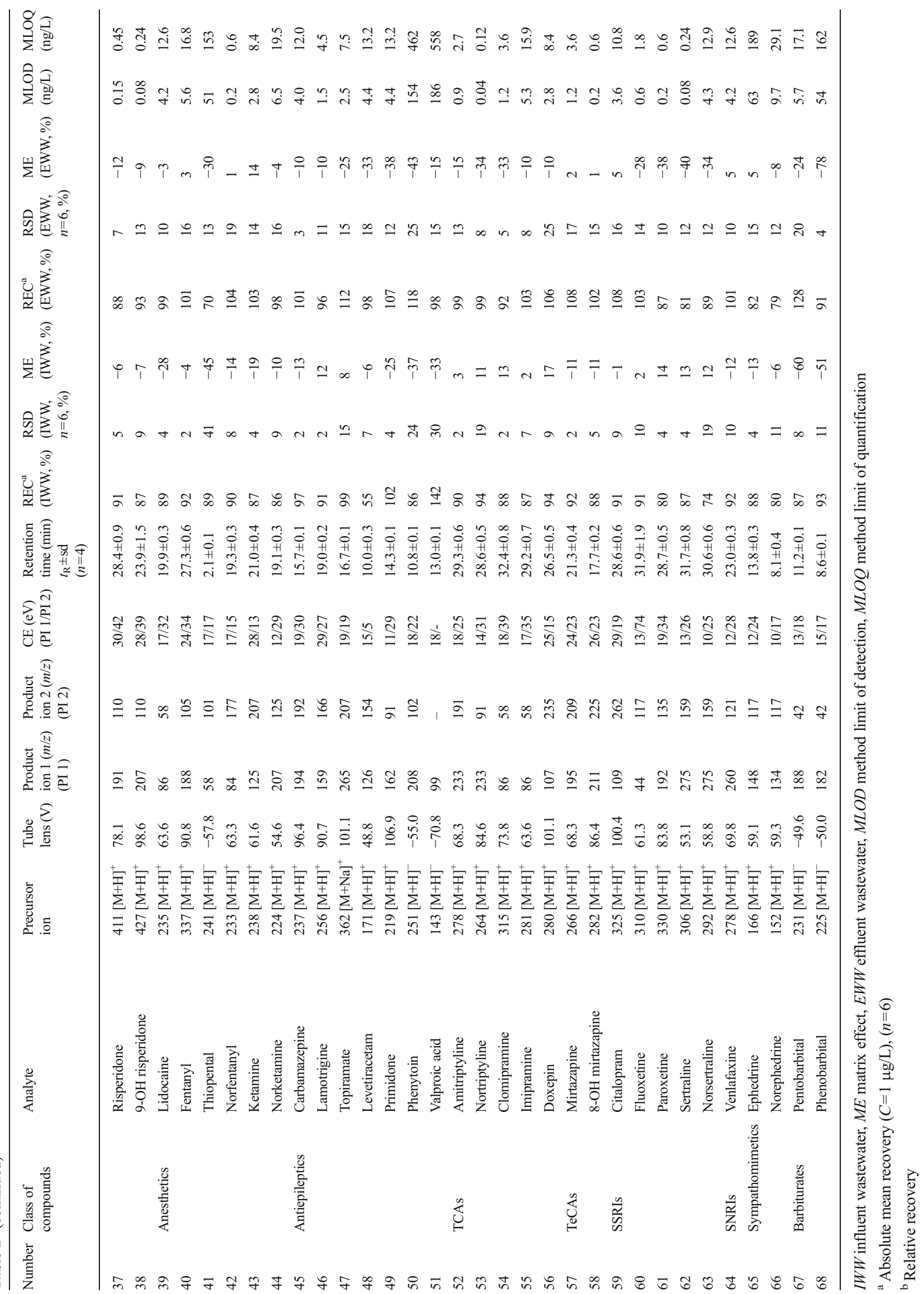


exception in this group and are not charged. Other families of compounds, including antiepileptics, cannabinoids, and barbiturates, are also neutral at this $\mathrm{pH}[45,49]$. In order to deal with these significantly different physicochemical properties, it was necessary to reach a compromise which provided good recovery rates for most compounds.

Two types of poly-vinyl-3-piperidone-divinylbenzene polymeric SPE cartridges, Strata-X (with neutral polymeric sorbent), and its sulfonated analog, Strata-XC, were tested for the determination of the target compounds. Strata-XC has been successfully previously employed to pre-concentrate basic illicit drugs from wastewater [30, 34, 43], whereas Strata-X has never been used for the determination of drugs. Strata-X cartridges provided absolute recoveries above $80 \%$ for most compounds (60), only three compounds showed recoveries between 60 and $79 \%$, and six compounds lower than $59 \%$ (experiments performed with IWW). EME, an important cocaine metabolite, was not possible to retain with this cartridge. Strata-XC cartridges provided absolute recoveries above $80 \%$ for 56 compounds (including EME), between 60 and $79 \%$ for 6 compounds, and lower recoveries than $59 \%$ for 6 compounds, as it is shown in Fig. 3. Figure 4 shows a comparison of the obtained absolute recoveries for some relevant selected compounds (IWW). Most of them showed similar recovery rates with both approaches. It was also observed that cannabinoids showed low but very reproducible recoveries (relative standard deviation (RSD) values $<10 \%$ for all compounds) using both cartridges. For these compounds, recoveries were higher with Strata-XC (although with no significant statistical differences taking into account

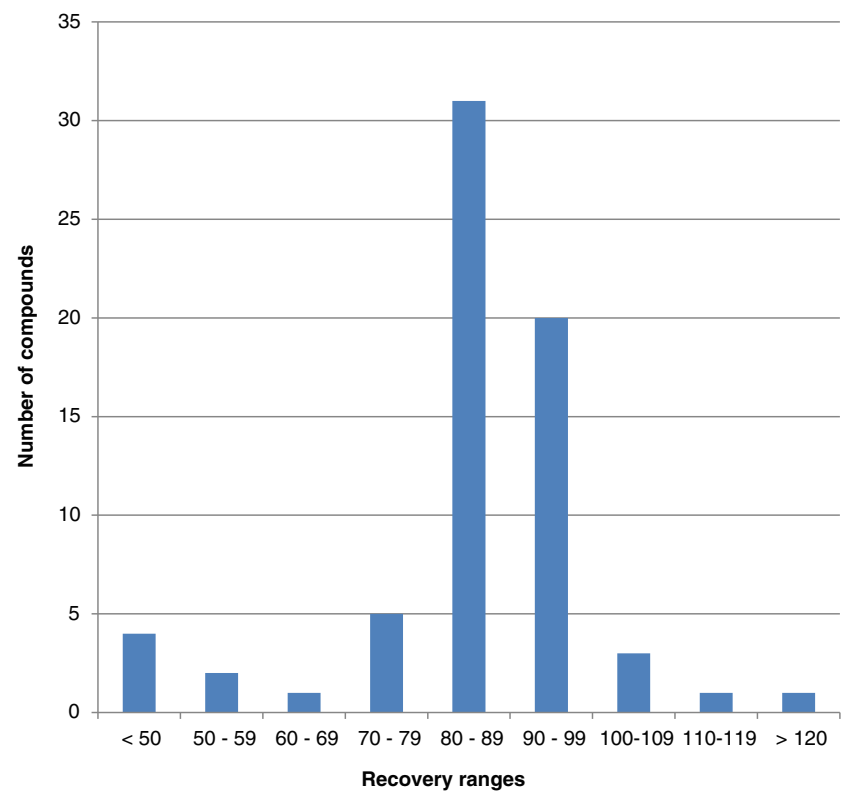

Fig. 3 Recoveries organized in ranges for the target analytes using Strata-XC (influent wastewater: spiked concentration, $1 \mu \mathrm{g} / \mathrm{L}$; sample volume, $50 \mathrm{~mL}$ ) the RSD values) because the two nonaromatic fused rings of the cannabinoids as well as the alkyl side chain of the THC$\mathrm{COOH}$ structure are able to interact more strongly through dispersive and pi-pi interactions with the divinylbenzene nucleus of Strata-XC in comparison with Strata-X [50]. Detection of EME, which was only possible using Strata$\mathrm{XC}$, is of capital importance because it is an important metabolite, along with benzoylecgonine (BECG), of cocaine, and it is necessary in order to obtain solid consumption data of this drug. The proposed final cartridge for further application of the method after a certain compromise was Strata-XC. This cartridge showed better hydrophobic as well as polar retention characteristics, especially for EME and other polar metabolites, compared to the neutral polymer with the same backbone structure, Strata-X. Moreover, it provides lower matrix effect for most of the compounds studied.

Vacuum pressure is also an important parameter during the SPE process, especially at high pressures. It was observed that when applying more than $10 \mathrm{psi}$, different compounds, including morphine, 6-MAM, buprenorphine, EDDP, OC, 8$\mathrm{OH}$ mirtrazepam, nordiazepam, and flunitrazepam, showed significantly lower recoveries than the ones obtained applying $8 \mathrm{psi}$, which was determined as the optimum value to perform the SPEs.

\section{Optimization of liquid chromatography}

Different experiments were carried out in order to evaluate the analyte retention times, peak shapes, and sensitivity during the chromatography procedure. Three different LC columns were evaluated: Kinetex PFP reversed-phase fused-core column $(50 \mathrm{~mm} \times 2.10 \mathrm{~mm}, 1.7 \mu \mathrm{m})$, Kinetex reversed-phase fusedcore $\mathrm{C} 18$ column $(100 \mathrm{~mm} \times 2.10 \mathrm{~mm}, 2.6 \mu \mathrm{m})$, and Kinetex C18-XB column $(100 \mathrm{~mm} \times 2.10 \mathrm{~mm}, 2.6 \mu \mathrm{m})$, all from Phenomenex ${ }^{\mathrm{TM}}$. Different mobile phases and gradient conditions were also tested. Stronger retention due to the $p-p$ bonding was observed when using a PFP column compared with $\mathrm{C} 18$ and $\mathrm{C} 18-\mathrm{XB}$ columns for all the studied compounds. This effect was especially pronounced for opiates and opioids, amphetamines, antipsychotics, and antidepressants. On the other hand, cannabinoids, antiepileptics, and benzodiazepines were eluted faster with a PFP column compared with $\mathrm{C} 18$ or $\mathrm{C} 18-\mathrm{XB}$ columns. Antidepressants and benzodiazepines showed similar chromatographic characteristics with the three columns. As it is shown in Fig. 5, amphetamines presented peak area asymmetry using the $\mathrm{C} 18$ and C18-XB columns, with a strong peak fronting effect (Fig. 5b, c). The PFP column was more appropriate for these compounds, providing higher sensitivity and more symmetric peaks for amphetamines and compounds containing $-\mathrm{NH}_{2}$ or -NH- groups (Fig. 5a). The PFP column provided also high resolution and sensitivity, along with minimal peak asymmetry, for sympathomimetics (Fig. 5d). In contrast, C18 columns 
Fig. 4 Influence on the recovery efficiency of the different cartridges tested (Strata-X and Strata-XC) for selected compounds (influent wastewater: spiked concentration, $1 \mu \mathrm{g} / \mathrm{L}$; sample volume, $50 \mathrm{~mL}$ )

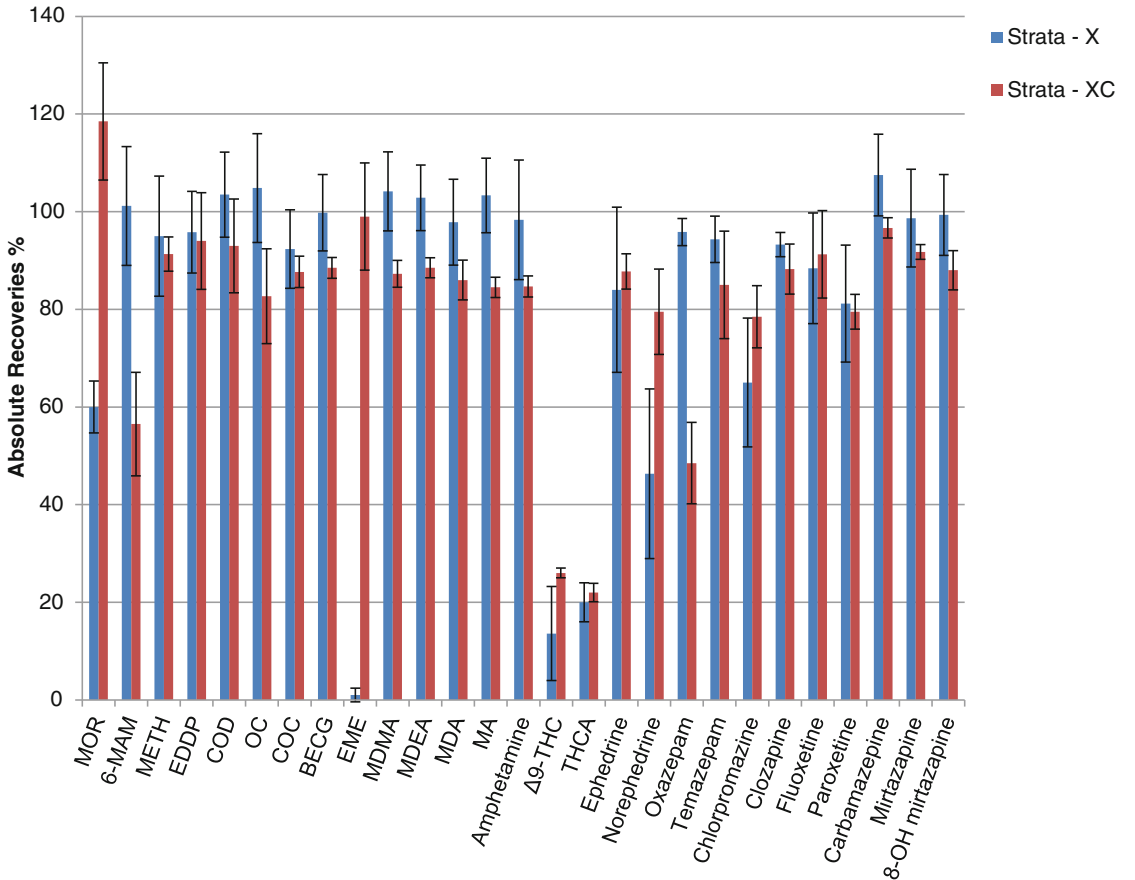

led to peak tailing and low sensitivity for these compounds (Fig. 5e). Similar behavior was also observed for opiates and opioids. In conclusion, better results were obtained using the PFP column for most compounds, and it was the chosen column to perform further experiments. However, it is necessary to point out that the comparison was carried out with columns of different lengths and particularly different particle diameters that may have an influence in the performance of the chromatography. Some exceptions were the mid-polar antipsychotics olanzapine and norclozapine, which showed better peak shape with the $\mathrm{C} 18$ columns. However, sensitivity was further improved for these compounds by adjusting the gradient of the optimum mobile phase.

Several mobile-phase combinations were tested for the PI mode experiments, using $\mathrm{MeOH}$ and $\mathrm{ACN}$ as organic solvents. Different concentrations of formic acid (organic modifier) were also tested to determine the best conditions to obtain a maximum peak resolution and little signal suppression. Electronic Supplementary Material Fig. S1 shows how the different mobile phases affect the peak area of the studied analytes. Different behaviors towards the different conditions were observed. In general, the addition of formic acid increased the peak areas of many compounds (e.g., above $50 \%$ in the case of benzodiazepine), because it facilitates the positive ionization. Formic acid also significantly improved the peak shapes for most analytes. For most families of compounds (opioids and opiates, cocaine compounds, antidepressants, anesthetics, benzodiazepines, amphetamine compounds, antiepileptics), methanolic mobile phases provided better results, improving the chromatographic resolution compared to ACN. Some exceptions can be found in the SSRI antidepressants fluoxetine and paroxetine and the antipsychotics family, where ACN improved the peak shape of all the compounds in the group. Finally, reaching a compromise, the best results were obtained using $\mathrm{MeOH}$ and Milli-Q water (both containing $0.05 \% \mathrm{v} / v$ formic acid), allowing a satisfactory elution for all the studied compounds (Electronic Supplementary Material Fig. S1). Retention times for all the compounds are detailed in Table 2. In the NI mode, the best chromatographic conditions were achieved using $\mathrm{MeOH}$ and Milli-Q water without the use of formate buffer or formic acid. Column operational temperature (column oven) was also optimized. Different temperatures (from 25 to $50{ }^{\circ} \mathrm{C}$ ) were tested, although in general, no significant effects were observed in the sensitivity of the analytes. A temperature of $25{ }^{\circ} \mathrm{C}$ proved to be the optimum column temperature since some compounds (e.g., methadone, EDDP, oxycodone, cocaine, clobazam, risperidone, imipramine, mirtazapine, venlafaxine, fluoxetine, sertraline, and norsertraline and norephedrine among others) showed better sensitivity.

\section{MS/MS parameter optimization}

MS/MS operational parameters were determined and optimized by direct injection (infusion) experiments of individual standard solutions. The first issue was to address the selection of the ionization mode (ESI) that enhances the formation of the protonated or deprotonated adduct in the ionization source for each target compounds and IS. In general, most compounds showed more efficient ionization in the PI mode, 
Fig. 5 Comparison of chromatograms for amphetamines and sympathomimetics using different columns. In all cases, the mobile phase consists in MeOH/Milli-Q water, $0.05 \% v / v$ formic acid. a Amphetamines with PFP column. b Amphetamines with $\mathrm{C} 18$ column. c Amphetamines with C18-XB column. d Sympathomimetics with PFP column. e Sympathomimetics with C18 column. f Sympathomimetics with C18-XB column
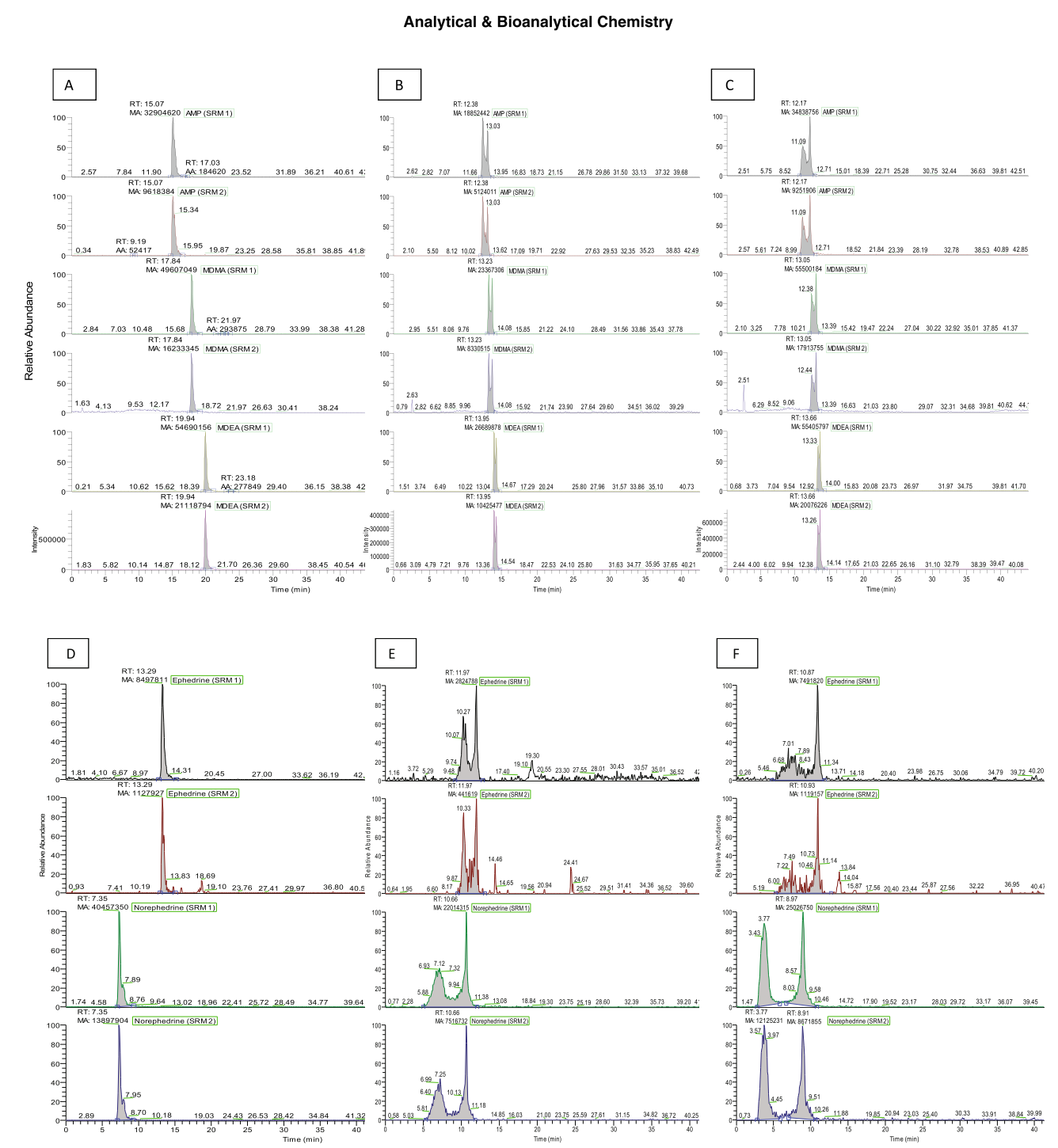

although for some of them, ionization in both modes is possible (e.g., THC and derivatives and topiramate). Thus, the resulting method includes 63 compounds in the PI mode and 5 in the NI mode. For all the compounds determined in the PI mode, the precursor ion was $[\mathrm{M}+\mathrm{H}]^{+}$except for topiramate. For this compound, the intensity of the protonated adduct was very low, and the intense and reproducible sodium adduct $[\mathrm{M}+\mathrm{Na}]^{+}(\mathrm{RSD}<15 \%)$ was selected to perform the analysis. In the NI mode, all the precursor ions corresponded to the deprotonated molecule $[\mathrm{M}-\mathrm{H}]^{-}$. Topiramate was also ionized in the NI mode, but the signal was 25 -fold lower than that obtained in the PI mode. ESI conditions in both the PI and NI modes were obtained as a compromise using the optimum values for most compounds. These conditions are described in detail in Electronic Supplementary Material Table S2. Table 2 summarizes the optimized values of MS/MS parameters for the studied compounds. Tube lens voltages were optimized for each compound in order to enhance the formation of the corresponding pseudo-molecular ion, to enhance desolvation, and to prevent in-source fragmentation. The product ions (two for each compound) were identified; two SRM transitions per compound (quantification and confirmation) were selected, and collision energies were optimized. Fragmentation patterns of all the substances, including metabolites, were also studied. This information is detailed in the Electronic Supplementary Material Table S3.

\section{Method validation}

The performance of the method was evaluated under the optimized conditions in terms of linearity range, sensitivity, accuracy, repeatability, and ME. Instrumental analytical parameters, linearity ranges, correlation coefficients $\left(r^{2}\right)$, and intraday instrumental precision, expressed as RSD, were evaluated. The calibration curves obtained for the SRM transitions were linear for all the compounds in a wide range of 
concentrations, typically from 0.1 to $100 \mu \mathrm{g} / \mathrm{L}$ with $r^{2}>$ 0.9978 for all compounds. Good instrumental precision was obtained with RSD values in the range of 3-5\% (intraday precision).

The method was validated for effluent and influent wastewater samples. Table 2 summarizes the method performance parameters for each compound. Quantification was based on standard additions, and deuterated internal standards were used only for the quantification of those compounds in which isotopically labeled analogs were available. The use of deuterated analogs was imperative for THC and THCA due to significant losses during sample preparation.

Extraction recoveries for target compounds were determined $(n=6)$ at one concentration of $1.0 \mu \mathrm{g} / \mathrm{L}$ for both IWW and EWW, mainly because many compounds were already present in the samples, and a direct comparison of recoveries between the two matrices was possible. Recoveries were determined by comparing the concentrations obtained after the whole procedure (pre-extraction spike) with the initial spiking levels in matrix extract (post-extraction spike). As real samples already contained the target compounds, nonspiked samples were analyzed in order to determine their concentrations, which were afterwards subtracted from the spiked samples. Recovery values ranged from 80 to $120 \%$ for the more than $80 \%$ of target compounds in both IWW and EWW. In general, polar compounds showed high recovery values due to the ionic interactions with the sulfonic moiety of the Strata-XC cartridge sorbent. For cannabinoids, low recoveries were obtained due to the strong adsorption of these substances to the Strata-XC particles, and due to the pi-stacking interactions between their aromatic ring and the electron deficiency of the benzene ring attached to the sulfonic acid moiety. Some authors have also proposed sorption to glassware as a factor to explain the low recoveries of these compounds [51]. It significantly hinders the elution of these compounds, even if an acidic washing step is included. These difficulties have been previously reported by Huq et al. [50]. Recovery values for the compounds morphine, MDMA, benzoylecgonine, ketamine, cocaine, LSD, EDDP, methadone, fluoxetine, temazepam, and diazepam were higher than those reported by Bones et al. by using Strata-XC cartridges [26] and similar to those reported by Baker et al. by using Oasis MCX for amphetamines, benzodiazepines, opioids, and hallucinogenics [38]. The MLODs (lowest analyte concentration with a signal to noise $(\mathrm{S} / \mathrm{N})$ ratio of 3 ) and the MLOQ (concentration with $\mathrm{S} / \mathrm{N}$ ratio of 10 and imprecision lower than $20 \%$ ) were calculated by analyzing five times the lowest spiked concentration $(0.01 \mu \mathrm{g} / \mathrm{L}$ for most compounds). MLOD values, summarized in Table 2 , were less than or equal to $1.5 \mathrm{ng} / \mathrm{L}$ for 26 compounds. Significantly low MLOD values were achieved for nortriptyline, norclozapine, 9-OH risperidone, and sertraline, in the range of $0.04-0.08 \mathrm{ng} /$ L. Thirty-three compounds showed values between 1.5 and $10 \mathrm{ng} / \mathrm{L}$, and only seven analytes showed higher values, between 14 and $63 \mathrm{ng} / \mathrm{L}$, except phenytoin $(154 \mathrm{ng} / \mathrm{L})$ and valproic acid $(186 \mathrm{ng} / \mathrm{L})$. The MLOQs achieved are below the reported concentrations and allow a reliable quantification of these analytes in IWW and EWW samples.

The overall method repeatability was also evaluated by spiking wastewater samples with $1.0 \mu \mathrm{g} / \mathrm{L}$ of each compound $(n=6)$. For 65 investigated compounds, RSD values were below $21 \%$, and only for thiopental, phenytoin, and valproic acid that RSD values were higher.

A severe matrix effect in the quantitative LC-MS/MS analysis of drugs has been widely observed $[52,53]$. The ESI source is highly susceptible to other components present in the matrix, which may result in a signal suppression or enhancement. Matrix effect values are summarized in Table 2. In most cases, signal suppression was observed, and only 16 compounds out of 68 showed signal enhancement (from 2 to $17 \%$ ). Forty-four out of the 68 analyzed compounds showed matrix ionization suppression between -1 and $-28 \%$, and only eight compounds between -33 and $-60 \%$. The robustness of the method was also studied during the validation procedure, testing different injection volumes and introducing slight changes in the $\mathrm{pH}$ of the mobile phase, in the gradient setting and in the temperature. As it was observed, these changes did not influence significantly the retention times or the sensitivity. SRM chromatograms of all the compounds in a spiked wastewater sample are presented in Electronic Supplementary Material Table S5.

\section{Application to real samples}

The occurrence of the selected illicit and psychiatric drugs in IWW and EWW from five WWTPs located in Santorini Island was evaluated using the multi-residue method described above. Sampling details have already been described in the "Materials and methods" section. Concentration values of the target compounds analyzed are summarized in Fig. 6 and Electronic Supplementary Material Table S4. Both drugs of abuse and psychiatric drugs were detected in all the processed samples; 58 out of the 68 compounds were detected at least in one sample, and 32 out of the 68 compounds investigated were detected in all IWW and 22 in EWW with very different ranges. The compounds imipramine, thiopental, olanzapine, fentanyl, chlorpromazine, 6-MAM, MDA, MA, chlordiazepoxide, and nitrazepam were not detected in any sample.

Figure 6 shows the contribution of the different families of compounds to each sample of both IWW and EWW. The detailed concentrations are available in Electronic Supplementary Material Table S4. The high contribution of antiepileptics is highlighted, denoting the high use of these pharmaceuticals. Of particular interest are the high values for carbamazepine, levetiracetam, and valproic acid determined for both IWW and EWW, with concentrations above $6 \mu \mathrm{g} / \mathrm{L}$ in some cases. The high values of ephedrine in IWW (the only 


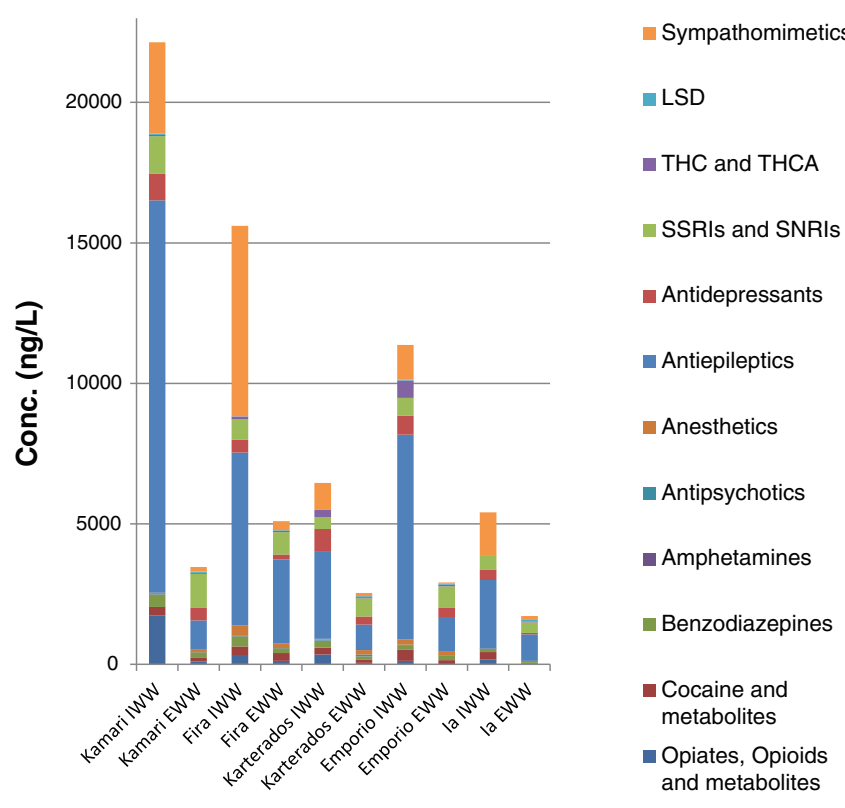

Fig. 6 Contribution of each family of compounds in the analyzed influent and effluent wastewaters

sympathomimetic that was detected) were also very relevant, with concentrations up to $6.7 \mu \mathrm{g} / \mathrm{L}$. Lower concentrations (but still significant) were detected in EWW for this compound. Antidepressants were also widely present in all the studied samples; the TCAs amitriptyline and doxepin and the TeCA mirtazapine showed the highest values, above $500 \mathrm{ng} / \mathrm{L}$ in some samples. Also, SSRIs and SNRIs were detected, citalopram and venlafaxine being the most relevant with values up to $733 \mathrm{ng} / \mathrm{L}$. These values are in agreement with those of previous reports from Spain and Canada [22, 54].

High consumption of the illicit drugs cocaine and THC was determined since high concentrations of the metabolites of these compounds were observed. BECG and EME and THCA were determined in all the WWTP locations, along with their parent compounds. LSD concentrations were very low. This fact can be explained: LSD doses are in the order of micrograms (instead of milligrams as for the other drugs), and also, LSD is intensively metabolized before excretion, giving as major excretion products LSD, nor-LSD, and OH-LSD, which was detected in relatively high concentrations in all samples $(22-54 \mathrm{ng} / \mathrm{L})$, indicating the consumption of this illicit drug. Low concentrations for MDMA and its metabolite MDEA were also determined.

Opiates, opioids, and their metabolites were detected in IWW of the most populated areas Kamari, Fira, and Karterados. However, no significant differences were observed in general between the levels in the different WWTPs, indicating a relevant consumption of these substances throughout the whole island.

For some compounds (e.g., OH-LSD, nordiazepam), it was observed that concentrations were higher in the EWW than in its corresponding IWW. This fact can be explained by the formation of unmeasured products of human metabolism and/ or transformation products (e.g., glucuronide conjugate, methylates, glycinates, etc.) that are passing through the plant converting back to the parent compounds. This can be considered as a reasonable assumption since the metabolites and some derivates of the mentioned compounds are well known (e.g., hydroxyl- and epoxy-derivatives of carbamazepine) [55]. However, it is important to consider that the samples analyzed were grab samples. Results indicated high consumption of these substances, but do not allow drawing sound longterm conclusions.

\section{Conclusions}

The present work reports the development and validation of a new analytical methodology for the simultaneous analysis of 68 compounds, including psychiatric drugs, illicit drugs, and some selected human metabolites (substances with a wide range of psychochemical properties) based on LCMS/MS using a PFP chromatographic column. A SPE step based on RP-cation exchange Strata-XC cartridges provided the best results. The multi-residue method proved to be highly sensitive (MLODs ranged from 0.04 to $10.0 \mathrm{ng} / \mathrm{L}$ for $86.7 \%$ of the compounds), allowing its use for monitoring the presence of the target analytes in real wastewater. Absolute recovery rates obtained ranged from 80 to $120 \%$ for most compounds (55 out of 68). Good results were obtained for EME, which is a significant metabolite of cocaine and necessary in order to obtain additional consumption data of this drug. The method was applied for the analysis of the selected drugs in wastewaters from five different networks located in Santorini Island (Cyclades Islands, Central Aegean Sea, Greece) during the peak tourist season. Results shows the high consumption of some illicit drugs including cocaine, methadone, ecstasy, LSD, and THC, confirmed by the presence of some metabolites such as EME, BECG, EDDP, MDME, LSD-OH, and THCA. The presence of high levels of psychiatric drugs including carbamazepine, valproic acid, levetiracetam, ephedrine, venlafaxine, oxazepam, amitriptyline doxepin, and oxazepam among others, was also assessed. Results also confirm the presence of most of these drugs in EWW at relevant concentrations, showing that the treatments applied in the selected WWTP are not effective enough for the complete removal of these compounds, and significant amounts of these substances are released to the marine environment in this case.

Acknowledgments This project was implemented under the Operational Program "Education and Lifelong Learning" and funded by the European Union (European Social Fund) and National ResourcesARISTEIA 624. 


\section{References}

1. Aldrich MR (2012) Antique cannabis book, medical cannabis: a short graphical history, http://antiquecannabisbook.com/chap1/ Shaughnessy.htm [Accessed on 12 Oct 2013]

2. EMCDDA (European Monitoring Centre for Drugs and Drug Addiction) (2012) Annual report: the state of the drug problem in Europe. http://www.emcdda.europa.eu/publications/annual-report/ 2012 [Accessed on 12 Jan 2014]

3. Kyriopoulos J, Tsiantou V (2010) Arch Hellen Med 27:834-840

4. Stuckler D, Basu S, Suhrcke M, Coutts A, McKee M (2009) Lancet 374:315-323

5. Daughton CG (2011) In: Castiglioni S, Zuccato E, Fanelli R (eds) Illicit drugs in the environment: occurrence, analysis and fate using mass spectrometry. Canada, Wiley

6. Van Nuijs ALN, Mougel JF, Taracomnicu I, Bervoets L, Blust R, Jorens PG, Neels H, Covaci A (2011) J Environ Monitor 13:10081016

7. Pal R, Megharaj M, Kirkbride KP, Naidu R (2013) Sci Total Environ 463-464:1079-1092

8. Jones-Lepp TL, Alvarez DA, Petty JD, Huckins JN (2004) Arch Environ Contam Toxicol 47:427-439

9. Boles TH, Wells MJM (2010) J Chromatogr A 1217:2561-2568

10. Zuccato E, Castiglioni S (2009) Phil Trans R Soc A 367:3965-3978

11. Zuccato E, Chiabrando C, Castiglioni S, Calamari D, Bagnati R, Schiarea S, Fanelli R (2005) Environ Health 4:14

12. Zuccato E, Castiglioni S, Bagnati R, Chiabrando C, Grassi P, Fanelli R (2008) Water Res 42:961-968

13. Daughton CG, Ruhoy IS (2009) Environ Toxicol Chem 28:24952521

14. Moore TJ, Glenmullen J, Furberg CD (2010) PLoS One 5:e15337

15. Binelli A, Pedriali A, Riva C, Parolini M (2012) Chemosphere 86: 906-911

16. Hummel D, Löffler D, Fink G, Ternes TA (2006) Environ Sci Technol 40:7321-7328

17. Postigo C, López de Alda MJ, Barceló D (2008) Anal Chem 80: 3123-3314

18. Postigo C, López de Alda MJ, Barceló D (2010) Environ Int 36:7584

19. Postigo C, López de Alda MJ, Barceló D (2011) Environ Int 37:49-55

20. Huerta-Fontela M, Galceran MT, Ventura F (2008) Environ Technol 42:6809-6816

21. Huerta-Fontela M, Galceran MT, Ventura F (2007) Anal Chem 79: 3821-3829

22. Gonzales Alonso S, Catala M, Maroto RR, Rodrigez Gill JL, De Miguel AG, Valcarcel Y (2010) Environ Int 36:195-201

23. Vazquez-Roing P, Andreu V, Blasco C, Pico Y (2010) Anal Bioanal Chem 397:2851-2864

24. González-Mariño I, Quintana JB, Rodriguez I, Cela R (2010) J Chromatogr A 1217:1748-1760

25. Bijlsma L, Sancho JV, Pitarch E, Ibáñez M, Hernández F (2009) J Chromatogr A 1216:3078-3089

26. Hernández F, Bijlsma L, Sancho VJ, Diaz R, Ibáñez M (2011) Anal Chim Acta 684:96-106
27. Boleda MR, Galceran MT, Ventura F (2007) J Chromatogr A 1175: $38-48$

28. Boleda MR, Galceran MT, Ventura F (2009) Water Res 43:11261136

29. Bueno MJM, Uclés S, Hernando MD, Fernández-Alba AR (2011) Talanta 85:157-166

30. Bisceglia KJ, Roberts AL, Schantz MM, Lippa KL (2010) Anal Bioanal Chem 398:2701-2712

31. Chiaia AC, Banta-Green C, Field J (2008) Environ Sci Technol 42: $8841-8848$

32. Metcalfe C, Tindale K, Li H, Rodayan A, Yargeau V (2010) Environ Pollut 158:3179-3185

33. Karolak S, Nefau T, Bailly E, Solgadi A, Levi Y (2010) Forensic Sci Int 200:153-160

34. Bones J, Kevin VT, Brett P (2007) J Environ Monit 9:701-707

35. Van Nuijs ALN, Pecceu B, Theunis L, Dubois N, Charlier C, Jorens PG, Bervoets L, Blust R, Meulemans H, Neels H, Covaci A (2009) Addiction 104:734-742

36. Van Nuijs ALN, Tarcomnicu I, Bervoets L, Blust R, Jorens PG, Neels H, Covaci A (2009) Anal Bioanal Chem 395:819-828

37. Kasprzyk-Hordern B, Dinsdale RM, Guwy AJ (2008) Water Res 42: 3498-3518

38. Bakera DR, Kasprzyk-Hordern B (2011) J Chromatogr A 1218: $1620-1631$

39. Kasprzyk-Hordern B, Dinsdale RM, Guwy AJ (2009) Environ Pol 157:1773-1777

40. Bijlsma L, Emke E, Hernández F, De Voogt P (2012) Chemosphere 89:1399-1406

41. Zuccato E, Castiglioni S, Tettamanti M, Olandese R, Bagnati R, Melis M, Fanelli R (2011) Drug Alcohol Depend 118:464-469

42. Berset JD, Brenneisen R, Mathieu C (2010) Chemosphere 81:859 866

43. Irvine RJ, Kostakis C, Felgate PD, Jaehne EJ, Chen C, White JM (2011) Forensic Sci Int 210:69-73

44. Terzic S, Senta I, Ahel M (2010) Environ Pollut 158:2686-2693

45. Chemicalize, http://www.chemicalize.org/ [Accessed on 12 Jan 2014]

46. Peel MC, Finlayson BL, McMahon TA (2007) Earth Syst Sci Discuss $4: 439-473$

47. van Nuijs ALN, Abdellati K, Bervoets L, Blust R, Jorens PG, Neels H, Covaci A (2012) J Hazard Mater 239-240:19-23

48. Baker DR, Kasprzyk-Hordern B (2011) J Chromatogr A 1218:80368059

49. Chemspider, www.chemspider.com [Accessed on 12 Jan 2013]

50. Huq S, Dixon A, Kelly K, Kallury KMR (2005) J Chromatogr A 1073:355-361

51. González-Mariño I, Quintana JB, Rodríguez I, Gonzáez-Díez M, Cela R (2012) Anal Chem 84:1708-1717

52. Kot-Wasik A, Debska J, Namiesnik J (2007) Trends in Anal Chem 26:557-568

53. Dams R, Huestis MA, Lambert WE, Murphy CM (2003) J Am Soc Mass Spectrom 14:1290-1294

54. Lajeunesse A, Gagnon C, Sauve S (2008) Anal Chem 80:5325-5333

55. Jelic A, Gros M, Ginebreda A, Cespedes-Sánchez R, Ventura F, Petrovic M, Barcelo D (2011) Water Res 45:1165-1176 